\title{
Biocontrol strategy of Diaphania Pulverulentalis targeting JHEH gene through Molecular cloning and Insilico analysis
}

\author{
Sriramadasu Kalpana ${ }^{1 *}$, Dadala Mary Mamatha ${ }^{1}$, K. Swetha Kumari ${ }^{1}$, Hephzibah A. R. Dadala ${ }^{2}$
}

${ }^{1}$ Department of Biosciences \& Sericulture, Sri Padmavati Women's University, India

${ }^{2}$ Department of Science, University Preparatory Academy, CA, USA

"Corresponding author: Sriramadasu Kalpana, Department of Biosciences \& Sericulture, Sri Padmavati Women's University, Tirupati, AP, 517502, India. Tel: +918772284588; Email: sriramadasu.kalpana@gmail.com

Citation: Kalpana S, Mamatha D.M, Swetha KK, Hephzibah ARD (2018) Biocontrol strategy of Diaphania Pulverulentalis targeting JHEH gene through Molecular cloning and Insilico analysis. Int J Genom Data Min 02: 117. DOI: $10.29011 / 2577-0616.000117$

Received Date: 05 December, 2017; Accepted Date: 26 December, 2017; Published Date: 03 January, 2018

\begin{abstract}
In insects, metamorphosis is controlled by interaction of endocrine agents mainly by Juvenile hormone and ecdysone. Juvenile Hormone Epoxide Hydrolase (JHEH), one of the major enzymes is involved in degradation of Juvenile hormone pathway and could be applied to control insects due to its irreversible reaction. The present work is focused on cloning of partial gene sequence and Insilico analysis of JHEH gene in Diaphania pulverulentalis $(D p)$ that is a serious insect pest on mulberry. A cDNA (800bps) encoding the partial JHEH was cloned from the mulberry leaf webber, Diaphania pulverulentalis. Partial $D p J H E H$ sequence contains an open reading frame encoding 244 amino acids with high degree of similarity to the reported insect JHEHs at NCBI. This is the kind of its first study on molecular cloning and sequence information of JHEH in Diaphania pulverulentalis.
\end{abstract}

Keywords: Cloning; In-silico analysis; Diaphania pulverulentalis; JH pathway; Juvenile hormone epoxide hydrolase

\section{Introduction}

Juvenile Hormone $(\mathrm{JH})$ is a sesquiterpenoid secreted by corpora allata in Insects. Juvenile Hormone plays an important role in physiology, development and reproduction in insect lifecycle. The JH titter in insects was regulated by JH biosynthesis and its degradation by specific enzymes [1]. JH levels are dramatically decreased at the final instar of larval growth, where the ecdysone levels are very high and responsible for pupation. The major enzymes involved in reducing and scavenging the $\mathrm{JH}$ before moult of last instar larval stage to pupa were Juvenile Hormone Esterase (JHE), Juvenile Hormone Epoxide Hydrolase (JHEH) and Juvenile Hormone Diol Kinase (JHDK). Formation of JH acid metabolite occurs by the hydrolysis of methyl ester of JH by JHE and hydration of epoxide moiety to JH diol is done by JHEH or JHEH acid diol will be formed by the simultaneous action of both the enzymes JHE and JHEH $[2,3]$.
Most of the research was concentrated on the mechanism and action of JHE in the JH degradation pathway. When compared to the JHE, very little is known about JHEH though it seems to be as critical as JHE in insect development. A microsomal epoxide hydrolase known as JHEH is a hydrolytic enzyme that belongs to the family $\alpha / \beta$ hydrolase. Due to the irreversible mechanism of JHEH on JH degradation pathway it could be applied to control insect pests [4]. JHEH genes have been characterized in many insects. In lepidopteran insect pests the JHEH genes are isolated from Manduca sexta [5], Ctenocephalides felis [6], Heliothis virescens [7], Trichoplusia ni [8], Spilarctia obliqua [9,10].

The insect pests attacking the silkworms and its host plant has increased and a new pest attack is being identifying regularly. These pests cause severe damage to the silkworm host plant and economic loss of Sericulture industry. Recently leaf defoliators become a serious threat to seri-farmers as they voraciously feed on young leaves of mulberry which are more important for chawki rearing. Among them Diaphania pulverulentalis (Hampson), commonly called as Leaf roller, has been observed as a severe pest on mulberry since 1995 in Karnataka state [11] that causes a major 


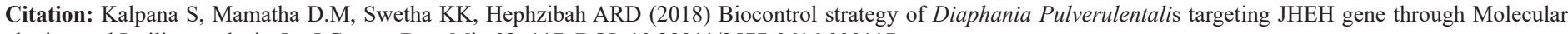
cloning and Insilico analysis. Int J Genom Data Min 02: 117. DOI: 10.29011/2577-0616.000117

damage to Mulberry tender leaves. Inhibition of JH degradation pathway causes changes in the $\mathrm{JH}$ titer leads to disturbances in physiology of Insects. JHEH is one of the enzymes involved in the removal of $\mathrm{JH}$ from the insects. In this paper, the cloning of the active sites and functional regions of JHEH sequence related with metamorphosis of last instar larvae in mulberry leaf webber - Diaphania pulverulentalis is reported. The obtained partial sequence of JHEH gene was analyzed by Insilico studies.

\section{Materials and Methods}

\section{Insect Rearing}

The larvae of Diaphania pulverulentalis of Mulberry were collected from infested mulberry gardens of surrounding villages of Chittoor District, Andhra Pradesh, India and rearing has done under optimum laboratory conditions. The larvae were fed with fresh V-1 variety mulberry tender leaves. They were reared in insect cage under controlled conditions $\left(24+1{ }^{\circ} \mathrm{C}, 75+5 \% \mathrm{RH}\right)$ and a photoperiod of 16:8 [12]. The cuticles of day 2, 3 and 4 of fifth instar larvae were dissected and preceded for the RNA isolation.

\section{RNA isolation}

The total RNA was extracted from cuticles of second, third and fourth days of fifth instar larvae of Diaphania pulverulentalis by TRIzol (Invitrogen) method. The tissues were weighed and frozen in liquid nitrogen. The tissue was homogenized with ceramic mortar and pestle. $1 \mathrm{ml}$ of TRIzol per $100 \mathrm{mg}$ of tissue was added and left for $30 \mathrm{~min}$ in room temperature until the sample (Tissue sample + TRIzol reagent) becomes liquefied. The samples were centrifuged at $12,000 \mathrm{rpm}$ for $10 \mathrm{~min}$ at $4^{\circ} \mathrm{C}$. Later, the supernatant was transferred into new tube and added $0.2 \mathrm{ml}$ of chloroform per $1 \mathrm{ml}$ of TRIzol. It was mixed vigorously and centrifuged at 12,000 rpm for $15 \mathrm{~min}$ at $4^{\circ} \mathrm{C}$. The aqueous layer was carefully separated and added $0.5 \mathrm{ml}$ of Isopropanol. It was incubated in $-20^{\circ} \mathrm{c}$ for $50 \mathrm{~min}$. The precipitated RNA was collected by centrifugation at $12,000 \mathrm{rpm}$ for $10 \mathrm{~min}$ at $4^{\circ} \mathrm{C}$. The pellet was twice washed with $75 \%$ ethanol and allowed it for partial drying. The partial dried RNA pellet was dissolved in $40 \mu \mathrm{l}$ of DEPC treated water. The quality and quantity of RNA was measured at 260/280 and 260/230 $\mathrm{nm}$ absorbance ratios with a spectrophotometer Nano Drop and was viewed by performing 2\% Agarose gel electrophoresis (Sigma Low EEO). The RNA samples were stored at $-80^{\circ} \mathrm{C}$.

\section{First Strand cDNA synthesis}

$2 \mu \mathrm{g}$ of total RNA sample was used to synthesize the single stranded cDNA. Oligo dT primer $(50 \mu \mathrm{M})$, dNTP mixture $(10$ $\mu \mathrm{M})$ and RNase free water were added to the total RNA and made up the volume to $10 \mu \mathrm{l}$. It was incubated for $5 \mathrm{~min}$ at $65^{\circ} \mathrm{C}$ and was immediately chilled on ice. To the above reaction mixture $5 X$ Prime script buffer, RNase inhibitor (40U/ $\mu 1)$, Prime Script
Reverse Transcriptase (200U/ $\mu 1$, Clontech) were added and made up to the volume $20 \mu \mathrm{l}$. The reaction mixture was mixed properly by vigorous shaking and incubated it for $10 \mathrm{~min}$ at $30^{\circ} \mathrm{C}$ followed by $60 \mathrm{~min}$ at $42^{\circ} \mathrm{C}$. Finally, the Reverse Transcriptase enzyme was deactivated by incubating at $95^{\circ} \mathrm{C}$ for $5 \mathrm{~min}$. The sample was cooled on ice. Working condition of cDNA was checked by PCR with 18s rRNA (internal control primers). The cDNA samples were stored in $-20^{\circ} \mathrm{C}$.

\section{Primer design}

The DpJHEH primers were designed based on its homologous sequences from Spodoptera litura, Manduca sexta, Heliothis virescens, Spilarctia obliqua, Helicoverpa armigera and Trichoplusia ni. Primer-Blast and Primer3 tools were used to check the presence of self complementarity, hairpin formation and dimerization.

\section{Amplification of $D p J H E H$ sequence}

A pair of degenerate primers (148F: 5' TNGAYMNTRAHGMGTGGTGGG 3', JHEH6R: 5 CCAAYWGTRTCAGGYTTSGTRGC 3') were used in the amplification of JHEH gene in Diaphania pulverulentalis. The PCR has been run on automated Master cycler Nexus (Applied Biosystems) using Platinum TM PCR super mix high fidelity. The volume of reaction mixture was $50 \mu \mathrm{l}$ having $45 \mu \mathrm{l}$ of Platinum TM PCR high fidelity mixture, $0.5 \mu \mathrm{l}$ each of $200 \mu \mathrm{M}$ forward primer (148F), reverse primer (JHEH6R), $1 \mu \mathrm{l}$ of cDNA and $3 \mu \mathrm{l}$ of PCR grade water. Gradient PCR was performed. The PCR conditions used were $2 \mathrm{~min}$ at $94^{\circ} \mathrm{C}, 40$ cycles of $15 \mathrm{sec}$ at $94^{\circ} \mathrm{C}, 30 \mathrm{sec}$ at $46-$ $51^{\circ} \mathrm{C}, 1 \mathrm{~min}$ at $68^{\circ} \mathrm{C}$ and $2 \mathrm{~min}$ at $68^{\circ} \mathrm{C}$.

The amplified products of expected size were analyzed on 1.5\% Agarose gel (Sigma, Low EEO) in 1X Tris-Acetate-EDTA buffer (40 mM Tris, $40 \mathrm{mM}$ acetic acid and $1 \mathrm{mM}$ ethylene diamine tetra acetic acid) stained with Ethidium bromide at $100 \mathrm{~V}$ for 60 min. The obtained bands were visualized by using Gel doc system. The generated expected size bands were cut from the gel, purified and eluted by PCR cleanup/DNA gel extraction kit (TAKARA) as per manufacturer's instructions. The purified products were sequenced by Sangers dideoxy nucleotide sequencing method. The chromatogram output was checked manually for minimizing ambiguities and base calling errors.

\section{Insilico analysis of the generated $D p J H E H$ gene}

Translated BLAST (blastx): The obtained nucleotide sequence similarity was determined based on BLAST search using the NCBI BLAST [13] server with comparison made to Non-redundant protein database by using blastx. The results were checked manually with respect to the percentage of identity and low e-values. The Partial sequence of $D p J H E H$ was submitted to NCBI ORF finder. 


\section{Multiple Sequence Alignment}

The nearest homologues of $D p \mathrm{JHEH}$ which were obtained in BLASTX results, retrieved from NCBI database are subjected to MAFFT [14] to identify the conserved regions. Further the Motif finder (www.genome.jp/tools/motif) and NCBI conserved domain search [15] was used to identify the biological significance sequence patterns and conserved domains in the DpJHEH ORF sequence.

\section{Phylogenetic Analysis}

Phylogenetic relatedness of $D p$ JHEH and JHEH sequences of six lepidopteran insect pests was done by Phylogeny.fr [16] to identify the evolutionary relationship among the JHEH sequences of insect pests. The tree was generated by Neighbour Joining method using Clustal format alignment.

\section{Structural analyses}

The obtained $D p J H E H$ protein sequence was subjected to PROTPARAM to explain the primary structural features like amino acid composition, Theoretical PI, Molecular weight, Instability index, grand average of hydropathicity.

Secondary structural analysis was done by SOPMA (Self Optimized Prediction Method with Alignment) [17] to identify the percentage of alpha helices, beta turns, extended strands and random coils in $D p J H E H$ protein sequence.

\section{Results}

The 2\% Agarose gel electrophoresis of total RNA showed high integrity bands of $28 \mathrm{~s}$ rRNA and $18 \mathrm{~s}$ rRNA in all the samples. The purity values of total RNA at 260/280 absorbance are within the range of 1.9 to 2.0. The gel picture of Total RNA is shown in the (Figure 1-4).

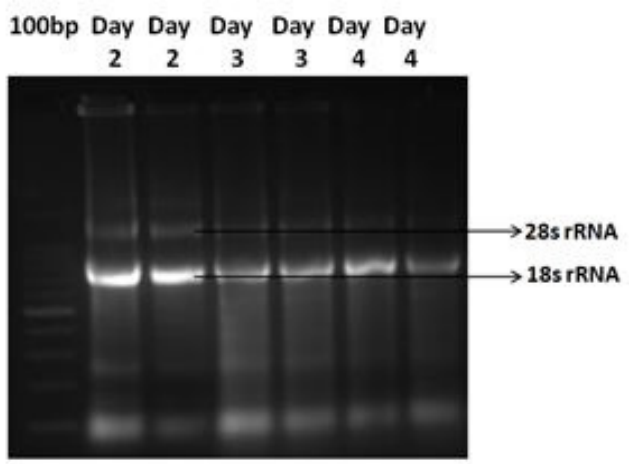

Figure 1: Agarose gel analysis of Total RNA samples of $D p$ fifth instar larvae.

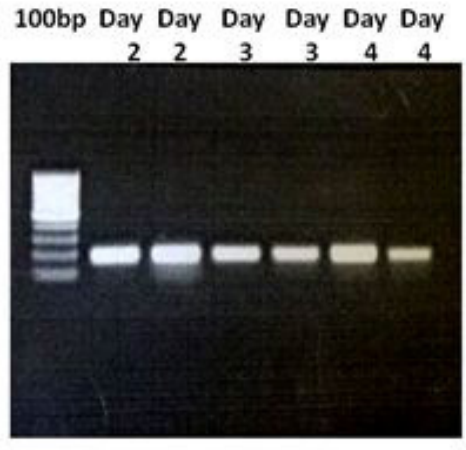

Figure 2: Agarose gel analysis of PCR reaction with $18 \mathrm{~S}$ rRNA control primers of $D p$ fifth instar larvae.

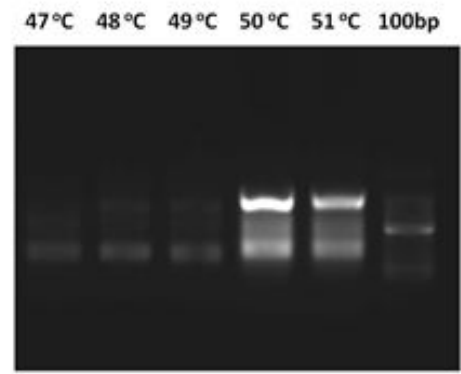

Figure 3: Gradient PCR $\left(46-51^{\circ} \mathrm{C}\right)$ amplification of Partial $D p J H E H$ gene using degenerate primers $148 \mathrm{~F}$ and JHEH6R.

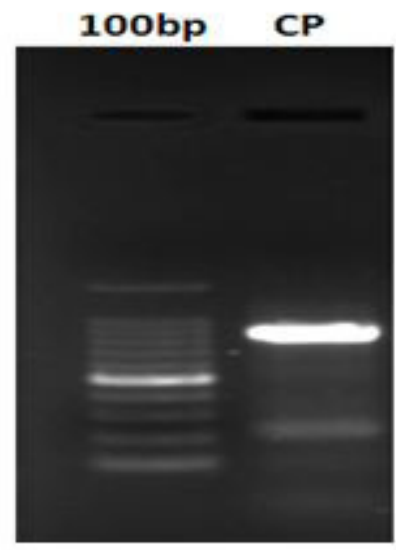

Figure 4: Column purification (CP) of amplified PCR product of Partial $D p$ JHEH gene using degenerate primers $148 \mathrm{~F}$ and JHEH6R. 
Citation: Kalpana S, Mamatha D.M, Swetha KK, Hephzibah ARD (2018) Biocontrol strategy of Diaphania Pulverulentalis targeting JHEH gene through Molecular cloning and Insilico analysis. Int J Genom Data Min 02: 117. DOI: 10.29011/2577-0616.000117

$>$ D.pulverulentails_JHEH_sequence

GGCCGAAAGACTGAAAGGACGAGTGGATAAGACCGTCAAGCCCTTCAAAATCAAGITIGAAGAATCGATGGTAA

CAGACCTTCGICAAAGACTAAAGAACCOCCGATCTITCGCGCCTCCTCTTGAAGGAGTGGGCTITGAGTATGGCTT

CAATACGAAGATCATCAAAGGCTGGCTGAGCTACTGGGCCGAGAAATACAACTTCGTCGAAAGGGAGAAGTTCT

GAACAAGTATCCTCAGTTCACTACTAACATTCAAGGATTGGATGTGCATTTCATAAGGGTGACGCCGCAGGTGTCG

GCAGGAGTTAAGTACTTCCTCTACTCCTGGTCCACG GCTGGCCAGGTCCGTCAGGGAGTTCTACGAGGCCATCC

CGCTTCTGACTCGCCAGACTCCAGGATACGACTTCGTGTIGGAGCTCATCGTTCCCAGTCTCCCTGGTTATGGTTAC

ACCGATGCTCCTGIGCGITCCGGCTTAGGCCTCCCACAAACAGCGGTAATCTICAGGAACCTGATGCACAGGCTGG

GCCACAAGAAGTTCTACGTGCAAGGAGGAGACTG GGGCGCGGGAGTCGGGGCTAGCATGGCCACOCTATTCCCG

GAAGACATCTTGGGATACCACAGCAACATGATIITGCACAGACCAAATGCTCAACTCTCATGTACATCTITGGGG

CTTACTTCCCCTCGTTCGTGGTGGAGTCAAAGCTGGCTGACCGCATGTACCCTATTTTTCATGGTICGCGTACATA

\section{CTTGAAGAGTTCGGCTACTTCCACCTGCAAGCCACCAAACCTG}

Figure 5: Amplified partial sequence (800bp) of DpJHEH gene.

The examination of cDNA working condition with 18S rRNA internal control primers was showed with well amplified bands with $200 \mathrm{bp}$ size. The gel picture was shown in the (Figure 2). The gradient PCR results inferred that expected amplicon size with 800 bps of $D p$ JHEH was amplified at $50^{\circ} \mathrm{C}$ annealing temperature. The PCR gel picture and purified product of PCR was shown in the (Figure $3 \&$ 4) respectively. The amplified partial $D p J H E H$ was shown in (Figure 5).

The partial $D p$ JHEH showed $71 \%$ homology with JHEH sequence of Spodoptera litura, $70 \%$ identity with Helicoverpa armigera, 69\% identity with Spodoptera exigua, 68\% with Amyelois transitella, $67 \%$ with Papilio xuthus and 66\% with Heliothis virescens. The first ten homologues of JHEH gene of Diaphania pulverulentalis based on blastx results were shown in the (Table 1).

\begin{tabular}{|c|c|c|c|c|c|}
\hline BLAST hit & $\begin{array}{l}\text { Max } \\
\text { score }\end{array}$ & $\begin{array}{l}\text { Total } \\
\text { score }\end{array}$ & $\begin{array}{l}\text { Query } \\
\text { coverage }\end{array}$ & E-Value & Identity \\
\hline Juvenile hormone epoxide hydrolase-like [Spodoptera litura $]$ & 417 & 417 & $99 \%$ & $1 \mathrm{e}-142$ & $71 \%$ \\
\hline Juvenile hormone epoxide hydrolase-like [Helicoverpa armigera] & 411 & 411 & $99 \%$ & $3 e-140$ & $70 \%$ \\
\hline Juvenile hormone epoxide hydrolase [Spodoptera exigua $]$ & 412 & 412 & $99 \%$ & $2 \mathrm{e}-140$ & $69 \%$ \\
\hline PREDICTED: juvenile hormone epoxide hydrolase-like [Amyelois transitella] & 406 & 406 & $99 \%$ & $2 \mathrm{e}-138$ & 68 \\
\hline $\begin{array}{c}\text { Hypothetical protein B5V51_6818 } \\
\text { [Heliothis virescens] }\end{array}$ & 399 & 399 & $99 \%$ & $2 \mathrm{e}-135$ & 68 \\
\hline
\end{tabular}


Citation: Kalpana S, Mamatha D.M, Swetha KK, Hephzibah ARD (2018) Biocontrol strategy of Diaphania Pulverulentalis targeting JHEH gene through Molecular cloning and Insilico analysis. Int J Genom Data Min 02: 117. DOI: 10.29011/2577-0616.000117

\begin{tabular}{|c|c|c|c|c|c|}
\hline PREDICTED: juvenile hormone epoxide hydrolase-like [Papilio xuthus] & 393 & 393 & $99 \%$ & $3 e-133$ & 67 \\
\hline $\begin{array}{l}\text { Microsomal epoxide hydrolase } \\
\quad[\text { Heliothis virescens }]\end{array}$ & 393 & 393 & $99 \%$ & $4 e-133$ & 66 \\
\hline PREDICTED: juvenile hormone epoxide hydrolase-like [Papilio polytes] & 392 & 392 & $98 \%$ & $1 \mathrm{e}-132$ & 67 \\
\hline $\begin{array}{l}\text { PREDICTED: juvenile hormone epoxide hydrolase-like isoform X2 [Papilio } \\
\text { machaon] }\end{array}$ & 391 & 391 & $99 \%$ & $2 \mathrm{e}-132$ & 67 \\
\hline $\begin{array}{l}\text { PREDICTED: juvenile hormone epoxide hydrolase-like isoform X1 [Papilio } \\
\text { machaon] }\end{array}$ & 390 & 390 & $99 \%$ & $3 e-132$ & 67 \\
\hline
\end{tabular}

Table 1: Blastx results against non-redundant protein database for partial $D p J H E H$ gene sequence.

The ORF sequence of partial DpJHEH generated by NCBI ORF Finder was showed in (Figure 6,7).

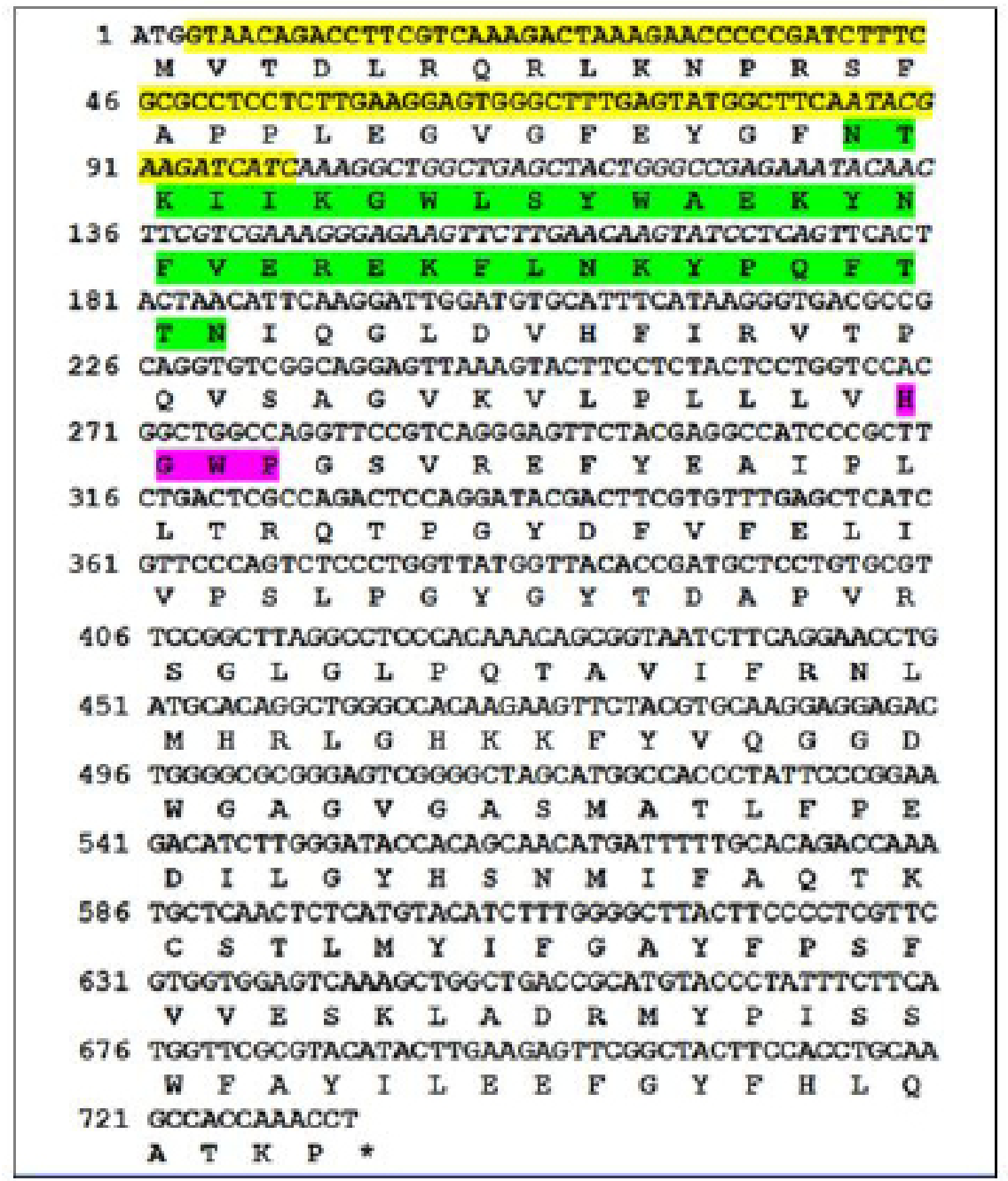

Figure 6: ORF sequence of $D p J H E H$ [HGWP motif-Pink; EHN domain (2-99) -Yellow; AbH domain (86-175) - Italic; $\alpha / \beta$ hydrolase domain (85-187)Green]. 
Citation: Kalpana S, Mamatha D.M, Swetha KK, Hephzibah ARD (2018) Biocontrol strategy of Diaphania Pulverulentalis targeting JHEH gene through Molecular cloning and Insilico analysis. Int J Genom Data Min 02: 117. DOI: 10.29011/2577-0616.000117

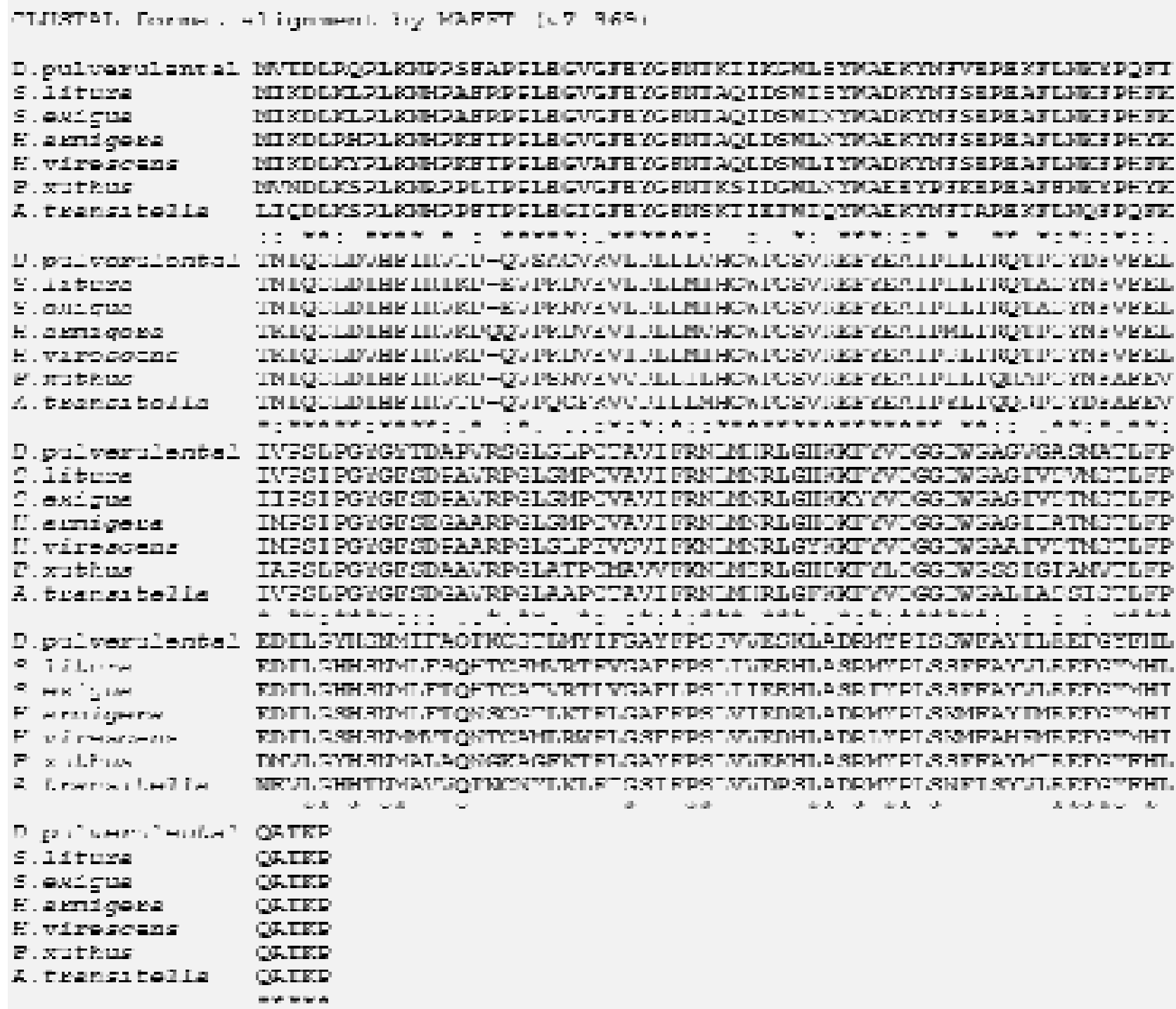

Figure 7: Multiple sequence alignment of $D p J H E H$ by MAFFT (Clustal format).

The comparison between the homologues of $D p J H E H$ by MAFFT showed the HGWP motif, Epoxide hydrolase N-terminus (EHN-Position 2-99), alpha/beta hydrolase fold (Abhydrolase_1-Position 85-187) and Alpha/beta hydrolase family (Abhydrolase_6Position 86-175). The multiple sequence alignment was shown in (Figure 7).

Three motifs Epoxide Hydrolase N-terminus (EHN-Position 2-99), alpha/beta hydrolase fold (Abhydrolase 1-Position 85-187) and Alpha/beta hydrolase family (Abhydrolase 6-Position 86-175) were identified in DpJHEH ORF sequence. The results of NCBI conserved domain search showed two functional domains EHN and Abhydrolase_1 super family in translated protein sequence of $D p J H E H$. The results were showed in (Figure 8A \& B). 
Citation: Kalpana S, Mamatha D.M, Swetha KK, Hephzibah ARD (2018) Biocontrol strategy of Diaphania Pulverulentalis targeting JHEH gene through Molecular cloning and Insilico analysis. Int J Genom Data Min 02: 117. DOI: 10.29011/2577-0616.000117
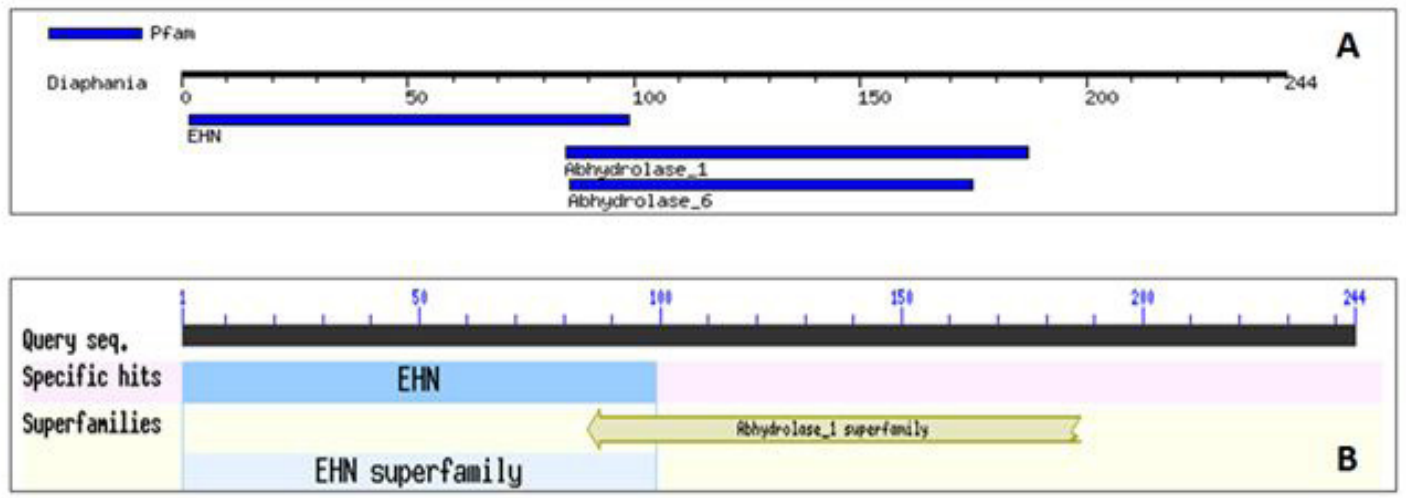

Figure 8: NCBI Conserved domain search for $D p J H E H$. A). Three motif regions identified in Partial $D p J H E H$ sequence by Motif finder. B). Two conserved domains regions identified in Partial $D p J H E H$ by NCBI conserved Domain search.

The generated phylogenetic tree showed that the partial $D p J H E H$ sequence is evolutionarily closer to the JHEH sequence of Amyelois transitella. The genetic divergence from the common ancestor can be observed in between JHEH sequences of Diaphania and Spodoptera species. The tree view of Phylogeny.fr was as shown in the (Figure 9).

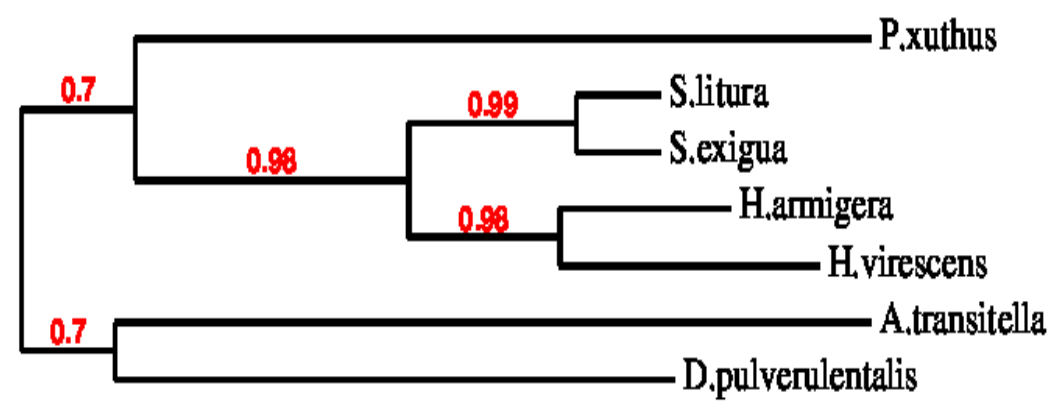

0.1

Figure 9: Phylogram of $D p J H E H$ sequence with other lepidopteran JHEH sequences.

The PROTPARAM output reveals that the DpJHEH contains 244 amino acids, 27822.19 molecular weight, 9.01 theoretical PI, 36.91 instability index, -0.007 GRAVY. The instability index of the sequence is below as 40 . Henceforth the $D p J H E H$ protein was predicted as stable. The protein sequence of $D p$ JHEH showed $33.6 \%$ of alpha helices, $19.26 \%$ extended strands, $9.84 \%$ beta turns and $37.30 \%$ random coils. The sequence and secondary structure representation (Figure 10A), the graphical representation of secondary structure (Figure 10B) and amino acid composition (Figure 10C) of $\mathrm{DpJHEH}$ are shown below. 
Citation: Kalpana S, Mamatha D.M, Swetha KK, Hephzibah ARD (2018) Biocontrol strategy of Diaphania Pulverulentalis targeting JHEH gene through Molecular cloning and Insilico analysis. Int J Genom Data Min 02: 117. DOI: 10.29011/2577-0616.000117

\section{MVTDLRQRLKNPRSFAPPLEGVGFEYGFNTKI I KGWLSYWAEKYNEVEREKF LUKYPQFTTNI QGLDVHE}

hhhhhhhhccccecccocccceecccccchhhhhhhhhhhhhhhhhhhhhhht tcceccccet tceeee IRVTPQVSAGVKVLPLLLVHGWPGSVREFYEAI PLTRQTPGYDEVELIVP SLPGYGYTDAPVRSGLGL

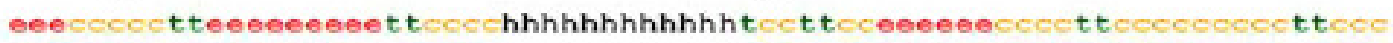
PQTAVI ERWLMHRLGHKKFYVQGGDWGAGVGASMAT LFPEDI LGYHSNMI FAQTKC STLMY FGAYFPSE

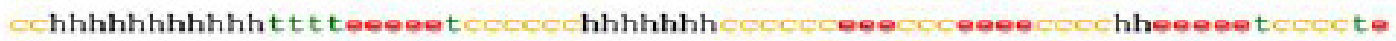
VVESKLADRMYPI SSWEAY I LEEEGYFHLQATKP

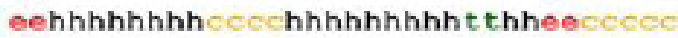
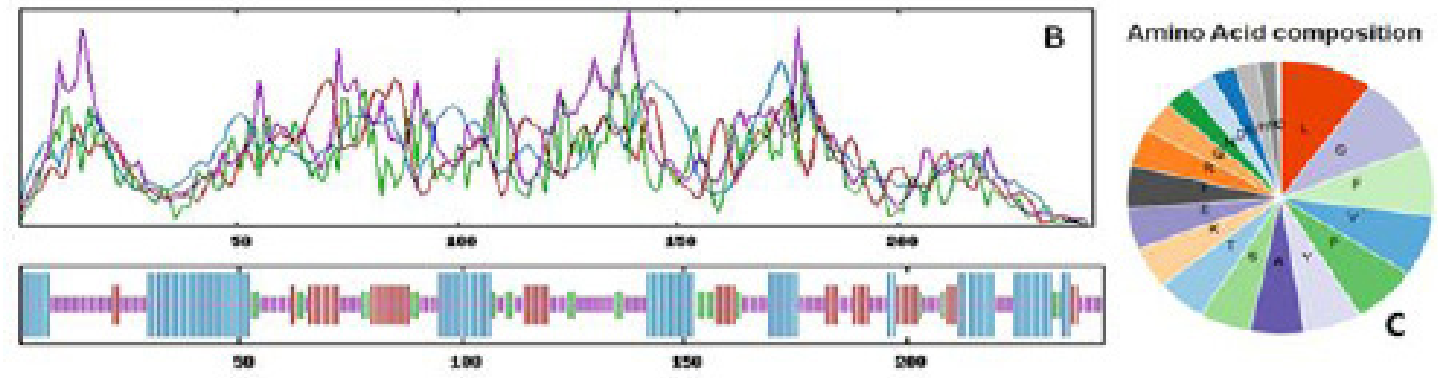

Figure 10: Secondary structural analysis of partial $D p J H E H$ protein sequence by SOPMA. A) Protein sequence with respective secondary structures. B) Graphical representation. C) Amino acid composition of $D p J H E H$.

\section{Discussion}

In insect life cycle, degradation of JH by JHE and JHEH plays a major role in JH titre during the time of secretion from Corpora Allata. Because of less information, there is no much research on JHEH. But due to irreversible mechanism on JH degradation pathway, it is very important to study the JHEH. To this point it could be applied to control the insect pests [1,2,18-20]. In Lepidoptera it has been suggested that $\mathrm{JH}$ acid act as a better substrate for $\mathrm{JHEH}$ than $\mathrm{JH}$ in the presence of $\mathrm{JH}$ binding protein. Until now the cloning studies of JHEH were done in few Lepidopterans namely Manduca sexta [5,17,21,22] Heliothis virescens [7] and Spilarctia obliqua [18]. In the present study we report the cloning of partial JHEH sequence of Diaphania pulverulentalis and its Bioinformatics analysis. Since it is a seriously identified pest on mulberry this is the first reported sequence in NCBI on JHEH gene of Diaphania pulverulentalis. The present research work reveals that the translated sequence of the amplified partial JHEH sequence showed the HGWP motif region which is an oxyanion hole-the most conserved region in all lepidopteran JHEH protein sequences. The high sequence similarity and identity of $D p J H E H$ with its nearest Lepidopterans concludes that these proteins could derive from a same ancestral gene and perform similar biological function. The genetic divergence have also be known and understood among different JHEH sequences of insect pests. The alignment of amino acid sequence of $D p J H E H$ reveals that they share common conserved regions. Insilico studies of partial $D p J H E H$ showed that its amino acid sequence contains three motif and two conserved domains that are mainly involved in catalytic functions. The Phylogenetic analysis revealed that the Amyelois transitella is the evolutionary closer to the DpJHEH. The amino acid composition, theoretical PI, Instability index was calculated. The instability index of the partial $D p J H E H$ is below 40 concluding that the protein is stable. The secondary structural features alpha helix, beta strand, extended strand and coils were identified in $D p J H E H$.

\section{Conclusion}

This is the first report on JHEH partial gene from Diaphania pulverulentalis. Insilico studies were done on the structural and functional features that could be used for further studies to elucidate the physiological and molecular functions. This work makes an important contribution toward understanding the features of JHEH gene in JH metabolism. Prediction of structural and functional features by Insilico studies facilitates the experimental analysis which leads to development of molecular biocontrol strategies like development of a recombinant biopesticides or even epoxide hydrolases could be identified as the best sources for getting used during the Xenobiotic metabolism in detoxifying the poisonous epoxides. The Insilico analysis was pre-requisite in predicting structural and functional features thereby facilitating the experimental analysis. 


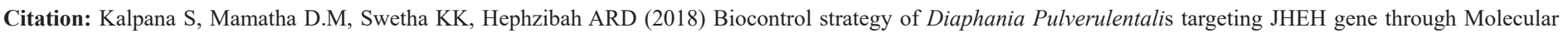
cloning and Insilico analysis. Int J Genom Data Min 02: 117. DOI: 10.29011/2577-0616.000117

\section{References}

1. Gilbert LI, Granger NA, Roe RM (2000) The juvenile hormones: historical facts and speculations on future research directions. Insect Biochemistry and Molecular Biology 30: 617-644.

2. Hammock, B.D. G.A.; Gilbert, L.I., ed., (1985) Regulation of juvenile hormone titer: Degradation. Comprehensive Insect Physiology, Biochemistry, and Pharmacology., New York: Pergamon Press., pp 431-472.

3. Share MR, Roe RM (1988) A partition assay for the simultaneous determination of insect juvenile hormone esterase and epoxide hydrolse activity. Anal Biochem 169: 81-88.

4. Hammock BD, Harshman LG, Philpott ML, Szekacs A, Ottea JA, et al. (1988) Strategies for the discovery of Insect control agents: exploitation of biomechanisms regulating insect development (edited by Steffens G.L. and Rumsey T.S.) 137-174.

5. Wojtasek H, Prestwich GD (1996) An insect juvenile hormone-specific epoxide hydrolase is related to vertebrate microsomal epoxide hydrolases. Biochem Biophys Res Commun 220: 323-329.

6. Keiser KC, Brandt KS, Silver GM, Wisnewski N (2002) Cloning, partial purification and in vivo developmental profile of expression of the juvenile hormone epoxide hydrolase of Ctenocephalides felis. Arch Insect Biochem Physiol 50: 191-206.

7. Shizuo G Kamita, Kohji Yamamoto, Mary M Dadala, Khavong Pha, Hammock BD, et al. (2012) Cloning and characterization of a microsomal epoxide hydrolase from Heliothis virescens. Insect Biochem Mol Biol 4: 219-228.

8. Harris S, Thompson DM, Linderman R, Tomalski M, Roe R, et al. (1999) Cloning and expression of a novel juvenile hormone-metabolizing epoxide hydrolase during larval-pupal metamorphosis of the cabbage looper, Trichoplusia ni. Insect Mol. Biol 8: 85-96.

9. M Hema, Dadala M Mamatha, K Swetha kumari (2013) Cloning and Insilico characterization of juvenile hormone epoxide Hydrolase gene of Spilarctia obliqua. International Journal of Agricultural Science and Research 3: 275-288.

10. Swetha Kumari K, Mary MD, Sriramadasu K, Dadala B (2015) Expression and characterization of recombinant Juvenile Hormone Epoxide Hydrolase of Spilarctia obliqua, a major pest in AgriSericulture. Mol Entomol 6.

11. Geethabai M, Marimadaiah B, Narayanaswamy KC, Rajgopal D (1997)
Anoutbreak of leaf-roller pest, Diaphania (Margaronia) pulverulentalis (Hampson) on mulberry in Karnataka. Geobios 16: 73-79.

12. Elham Yazdani, Jalal Jalali Sendi, Alireza Aliakbar (2013) Chemical composition, toxicity and physiological effects of essential oil of Rosemarinus officinalis on lesser mulberry pyralid, Glyphodes pyloalis Walker (Lepidoptera: Pyralidae). J. Crop Prot 2: 461-476.

13. Altschul SF, Gish W, Miller W, Myers EW, Lipman DJ, et al. (1990) "Basic local alignment search tool". J. Mol. Biol 215: 403-410.

14. Katoh K, Rozewicki J, Yamada KD (2017) MAFFT online service: multiple sequence alignment, interactive sequence choice and visualization.

15. Marchler-Bauer A, Derbyshire MK, Gonzales NR, Lu S, Chitsaz F, et al. (2015) "CDD: NCBI's conserved domain database". Nucleic Acids Res 43: 222-226.

16. Dereeper A, Audic S, Claverie JM, Blanc G (2010) BLAST-EXPLORER helpsyoubuilding datasetsforphylogeneticanalysis. BMCEvolBiol 10:8.

17. C Geourjon, G Deléage (1995) SOPMA: significant improvements in protein secondary structure prediction by consensus prediction from multiple alignments. Bioinformatics 11: 681-684.

18. Hammock BD, Quistad GB (1976) The degradative metabolism of juvenoids by insects, in The Juvenile Hormones, ed. By Gilbert LE. Plenum Press 374-393.

19. Roe, R.M., Venkatesh, K., 1990. Metabolism of juvenile hormone: degradation and titer regulation. In: Gupta, A.P. (Ed.), Recent Advances in Comparative Arthropod Morphology, Physiology, and Development, vol. 1. Rutgers University Press, New Brunswick, NJ, pp. 127-179.

20. Prestwich GD, Wojtasek H, Lentz AJ, Rabinovich JM (1996) Biochemistry of proteins that bind and metabolize juvenile hormones. Archives of Insect Biochemistry and Physiology 32: 407-419.

21. Debernard S, Morisseau C, Severson TF, Feng L, Wojtasek H, et al. (1998) Expression and characterization of the recombinant juvenile hormone epoxide hydrolase (JHEH) from Manduca sexta. Insect Biochem. Mol. Biol 28: 409-419.

22. Grieneisen ML, Kieckbusch TD, Mok A, Dorman G, Latli B, et al. (1995) Characterization of the juvenile-hormone epoxide hydrolase (JHEH) and juvenile hormone diol phosphotransferase (JHDPT) from Manduca sexta Malpighian tubules. Arch Insect Biochem Physiol 30: 255-270. 